\title{
Development of field-scale composite biorack constructed wetland - startup phase
}

\author{
Mitil Koli ${ }^{1}$ and Guru Munavalli ${ }^{2}$ \\ ${ }^{1}$ Department of Civil Engg., Walchand College of Engineering, Sangli, Maharashtra, India, \\ ${ }^{2}$ Department of Civil Engg., Walchand College of Engineering, Sangli, Maharashtra, India
}

\begin{abstract}
Constructed Wetlands (CW) with Biorack (BR) technology have been studied extensively in recent years with positive outcomes compared to conventional CWs. A field scale application of the same has been worked upon in the Composite Biorack Constructed Wetland $(\mathrm{CBCW})$ which is a secondary wastewater treatment facility of the Decentralized Wastewater Treatment System (DWTS) at Walchand College of Engineering, Sangli (M.S.), India. The CBCW is a multi-compartment system comprising of compartments with brickbats as supporting medium and BR Compartments (BRC) and has been in operation since May, 2018. The CBCW is vegetated with Typha angustifolia L. and Canna indica macrophytes. Being a unique CW, studies on difficulties aroused during the startup phase need to be addressed so as to arrive at proper troubleshooting techniques for future references. The paper addresses various issues, specifically growth of vegetation and their early wiltage in $\mathrm{CBCW}$, for a period of 150 days. Using alternative modes of plantation, it is concluded that vegetation acclimatization, proper suspension and grip of the bulb and roots of saplings in racks, fluctuating inflow of wastewater are few reasons influencing development of vegetation. An improvement by about $12 \%$ is obtained in COD removal in the latter period of study implying establishing of vegetation in $\mathrm{CBCW}$.
\end{abstract}

\section{Introduction}

Water scarcity has hit hard in most regions of developing countries like India. A huge iota of wastewater generated is disposed of untreated into surface water polluting the already scarce fresh water sources. With the emergence of new concepts in urban development such as Smart City in India, application of Decentralized Wastewater Treatment System (DWTS) can help curb this problem and treated wastewater can further be reused for nonpotable purposes subsequently reducing load on freshwater sources. DWTS are gaining popularity due to their low CAPEX and OPEX as well as a potential source of non-potable water. Constructed Wetlands (CWs) being one such system has gained popularity in the recent years owing to its simplicity and robust treatment performance [2] CWs consist of aquatic macrophytes planted on a bed of porous media on which wastewater is fed either horizontally or vertically. The macrophytes have an extensive root system having a significant ability to transport oxygen from the atmosphere to the roots which then disperses a portion of this oxygen into the rhizosphere. The micro-organisms thriving on the substrate utilize this oxygen and stabilize the organic matter applied on the CW. Plant species such as Phragmites sp. show higher organic removal along with heavy metals and could sustain environmental stresses [7,11] Use of multispecies vegetation instead of singular species such as Canna indica, Phragmites and Cyprus provide habitats for development of a varied microbial population on their root matrix and thereby aid in enhanced removal of pollutants. Few other pollutant removal mechanisms in a $\mathrm{CW}$ include sedimentation, filtration, nitrification-denitrification and phytoremediation. CWs are basically classified according to three criteria: i) hydrology (surface or subsurface flow) ii) type of plant species (emergent, submerged or free-floating) and iii) flow path (horizontal or vertical) [10]. The disadvantages of single type of wetland can be overcome with improvement in pollutant removal efficiencies by combining two or more CWs in a series or using technologies of two $\mathrm{CWs}$ into one thereby forming a composite or hybrid $\mathrm{CW}$. One of the major drawbacks of a $\mathrm{CW}$ is clogging of bed owing to high suspended solids. A more economical, compact and clogging free alternative to the conventional wetland system is Biorack (BR). A BR is a mesh of vertical PVC pipes arranged in a rectangular configuration within a tank. The pipes are provided with numerous perforations for liquid transportation. These BRs are involved in the treatment process by holding vegetation and providing abundant attachment site for microbial growth. BR system showed high rate of organic degradation of raw sewage effluent at lower HRT of 10h using Phragmites sp. [10] BRs were also found to be effective for wastewater with high total dissolved solids and heavy metal salts like cadmium, copper, nickel and zinc by using Phragmites sp. proving to be an ideal process for domestic wastewater treatment [11]. Vegetation such as Thalia dealbata planted in a BR was able to treat low concentration polluted river water due to higher nutrient removal rates of the plant species as well as high fineroot biomass [13]. [14] studied the performance of a BR fed with effluent through an anaerobic baffled reactor

* Corresponding author: guru.munavalli@walchandsangli.ac.in 
and using Typha sp. and Phragmites sp. The integrated system showed low capital investment and high COD, COD and $\mathrm{BOD}_{5}$ removal reaching $87 \%, 90 \%$ and $93 \%$ respectively at an HRT of $21 \mathrm{~h}$. BR was also efficient in $\mathrm{Cu}$ removal from $\mathrm{Cu}$-contaminated freshwater [7]. BR planted with multispecies of vegetation viz. Typha angustata and Canna indica showed a significant increase in organic degradation and enhancement of dissolved oxygen as compared to conventional Biorack [9]

The present paper addresses the various difficulties encountered in the startup phase of a field-scale Composite Biorack Constructed Wetland (CBCW) as well as different alternatives applied inorder to overcome them and aid for a smoother performance. The outcomes would contribute to existing knowledge on starting up a CW.

\section{Materials and methods}

\subsection{Source of wastewater}

CBCW is a secondary wastewater treatment facility of the DWTS at Walchand College of Engineering (WCE), Sangli (M.S.), India. The college is supplied with two major sources of water, i.e., through municipal water supply; treated in campus water treatment plant for potable use and open wells within the college campus for non-potable facilities. Wastewater generated through different the residential sections in campus such as the boys' and ladies hostels and staff quarters mainly comprise of septic tank effluent and greywater. The wastewater is first collected in a storage sump and then supplied for primary treatment through screens and Anaerobic Baffled Reactor (ABR) after which it enters the CBCW. This anaerobically treated wastewater forms the basis for the current study.

\subsection{CBCW details and operation}

Technically, DWTS in WCE is a multi-level natural wastewater treatment plant of $42 \mathrm{~m} 3 / \mathrm{d}$ capacity. Wastewater undergoes preliminary and primary treatment passing through screen chamber made up of coarse and fine screens for trapping large size floating matter and an underground chain of ABR for settlement of high suspended solids. The effluent from ABR enters CBCW for secondary treatment. $\mathrm{CBCW}$ is a brick masonry structure made up of twenty compartments comprising of an inlet chamber, alternate compartments having brickbat as supporting medium hereafter named as BSFW (Brickbat Subsurface Flow Wetland) and Biorack compartments (BRC), adsorption bed and an outlet chamber in a series with an effective wastewater depth of $0.64 \mathrm{~m}$. BRC are made up of Asbestos Cement (AC) sheets arranged so as to create space for plantation of vegetation. An adsorption bed made up of coarse aggregates and charcoal forms the final compartment before outlet chamber. The primary treated wastewater enters $\mathrm{CBCW}$ and flows in an upflow and downflow mode through all the compartments and treated effluent is then discharged into the outlet chamber after which it is pumped to a Vertical Flow Constructed Wetland (VFCW) for final treatment. The treated wastewater is then sent for land application. Fig. 1 shows a schematic line plan of CBCW.

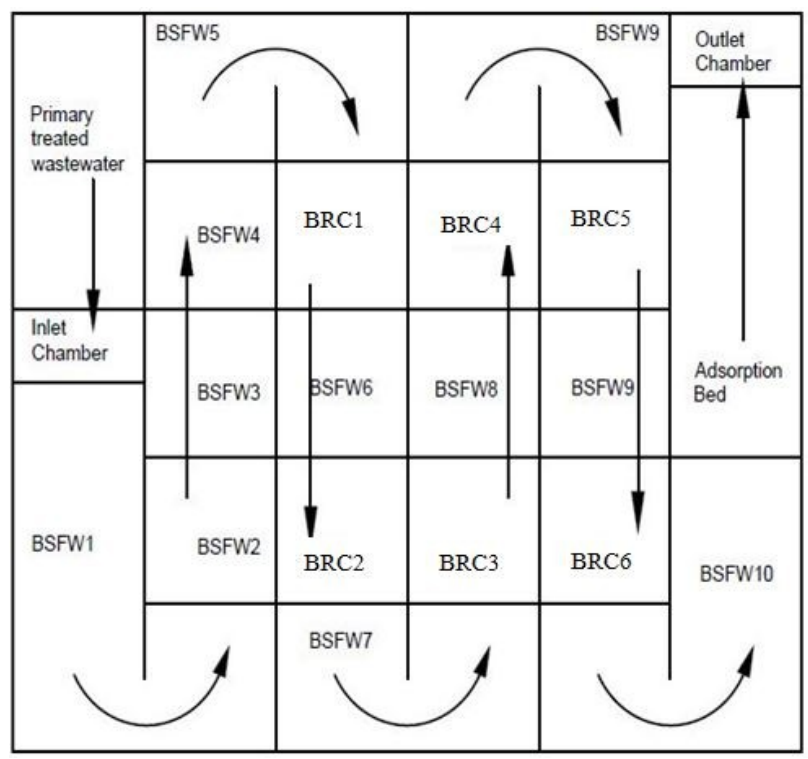

Fig. 1 Schematic line plan of $\mathrm{CBCW}$

\subsection{Vegetation details}

Normally, locally available wetland plants called macrophytes are used in a $\mathrm{CW}$ due to their abundance and easy availability if required in larger quantity. The vegetation used in CBCW was Typha angustifolia L. and Canna indica which are wetland macrophytes found in the streams and marshy lands of Sangli city. Typha angustifolia L. belongs to cattail (Typhaceae) family which has narrow leaves of width about $0.01 \mathrm{~m}$ and can grow upto $2 \mathrm{~m}$ tall. Advantage of using Typha angustifolia $\mathrm{L}$. is its effectiveness in removing pollutants such as carbonaceous organic matter, nitrogen and phosphorous. It also helps in enhancing dissolved oxygen level in wastewater due to oxygen leakage from root zone [5,6]. Canna indica is another widely found flowering plant with broad leaves and aesthetically pleasing flowers. The plant can grow upto $1 \mathrm{~m}-1.5 \mathrm{~m}$ tall and has an extensive growth rate. It is also effectual in nitrogen, phosphorus, carbonaceous matter removal as well has specific ability to uptake some of the heavy metals. The root matrix of this plant is extensive and also helps in increasing the dissolved oxygen of wastewater $[1,2,4,8,14]$. Since both plants have an extensive root matrix, if planted together they can provide habitat for diverse group of microbes leading to higher pollutant removal rates. These plants were carefully dug up using simple hand tools such that their roots and bulbs remained intact. The roots were then washed thoroughly 
to remove clay and any foreign matter and then planted in $\mathrm{CBCW}$ using different approaches.

\subsection{Monitoring of system}

CBCW was monitored from August, 2018 to December, 2018 considering it as the startup phase for this CW. Initially, flow measurement was carried out daily for a week in the month of August, 2018 between 6:00AM to 6:00PM so as to determine the fluctuations in the flow rate as well as determining the times for peak and least flow. Flow was measured by fill and draw method and was found to range between $532 \mathrm{~L} / \mathrm{h}$ and $7200 \mathrm{~L} / \mathrm{h}$ (least and peak flow respectively).

Initially, around 80 young Typha angustifolia L. and 30 Canna indica saplings were planted in $\mathrm{CBCW}$ in late July, 2018. These saplings were gripped together and placed in compartments consisting brickbats, by creating space between them such that root tips reached wastewater. Similarly, they were placed in AC sheet BRC as shown in Fig.2 (a). Ideally, the plants should develop shoots after a few days. However, most of the vegetation was observed to be wilting out particularly in BRC. This problem was addressed in month of September, 2018 by typing up stems of two plants together with a thread and then kept on the edges of AC sheet with their roots suspended in wastewater shown in Fig. 2 (b). After few days of this method of plantation, it was noticed that although very few number of saplings had developed new shoots, the majority of plants had begun to dry out Thus, no significant plant growth was achieved. Vegetation brought thenceforth from month of October, 2018 was then washed thoroughly and kept in a barrel filled with the freshwater and then subjected to series of diluted wastewater, continued till the plants were subjected to the wastewater which they would be exposed in $\mathrm{CBCW}$. Furthermore, these 3-4 saplings were gripped together into clean plastic water bottles assembled as a pipe cut on both sides and having perforations as shown in Fig. 3. These bottled plants were then inserted into the spaces between the AC sheets Biorack such that they remained suspended within those gaps. New shoots developed after a week of plantation in all the bottled plants with the parent plant wilting out. With daily observations it was found that the vegetation growth was significantly higher and new shoots developed within a week of plantation. It was noticed that around two to three new shoots were developed per Typha angustifolia L. plant and two new shoots per Canna indica using this approach of plantation. Harvesting of dried plants and replantation was done after every three weeks or once when large number of plants was wilted.

The effect of vegetation growth on removal of organic matter from $\mathrm{CBCW}$ was evaluated based on $\mathrm{pH}$, COD and $\mathrm{BOD}_{3}$. Wastewater samples were collected in clean $1 \mathrm{~L}$ plastic cans using grab sampling procedure from the inlet and outlet chamber. Frequency of sampling was mostly on a daily basis. The samples were then brought in Environmental Engineering Laboratory at WCE for analysis as per standard procedures set by APHA [3].

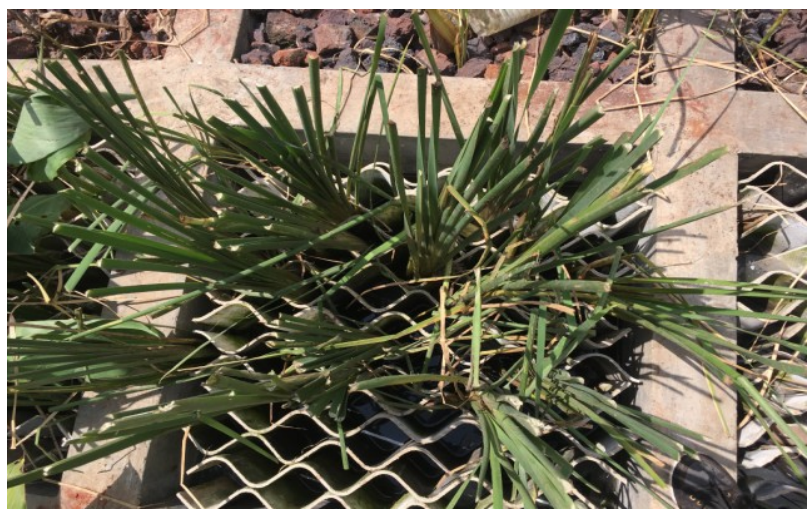

Fig. 2 (a) Photographic view of vegetation inside AC sheet Biorack

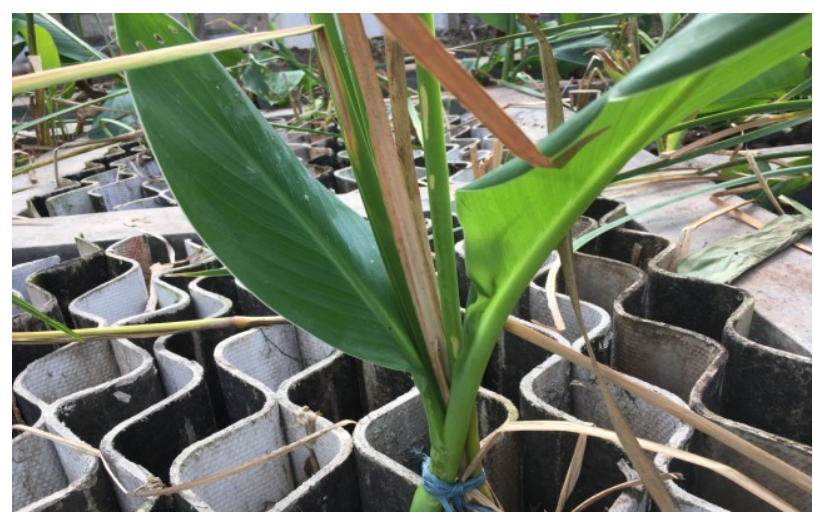

Fig. 2 (b) Photographic view of vegetation inside AC sheet Biorack

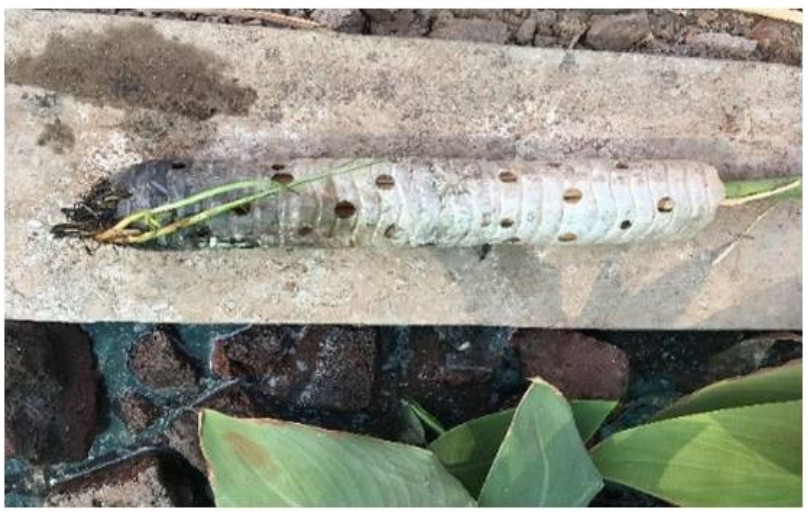

Fig. 3 Photographic view of vegetation in discarded bottle as Biorack

\section{Results and Discussions}

\subsection{Influent Characteristics}

A thorough chemical analysis of influent revealed the significant characteristics as mentioned in the Table 1. The $\mathrm{pH}$ values indicate slight alkalinity in wastewater conforming to the fact that the influent to $\mathrm{CBCW}$ is from 
ABR and supposedly has methanogenic activity taking place contributing to a slight high value of $\mathrm{pH}$. The values of $\mathrm{BOD}_{3}$ and $\mathrm{COD}$ indicate low strength of the influent along with $\mathrm{BOD}_{3} / \mathrm{COD}$ greater than 0.5 , which ascertains suitability of the influent into consideration to be suitable for biological treatment. $\mathrm{CBCW}$ being one such biological treatment unit can thus be an appropriate alternative for wastewater treatment.

Table 1. Characteristics of influent to $\mathrm{CBCW}$

\begin{tabular}{|c|c|c|c|}
\hline $\mathrm{pH}$ & $\mathrm{COD}$ & $\mathrm{BOD}_{3} @ 27^{\circ} \mathrm{C}$ & $\mathrm{TKN}$ \\
\hline- & $\mathrm{mg} / \mathrm{L}$ & $\mathrm{mg} / \mathrm{L}$ & $\mathrm{mg} / \mathrm{L}$ \\
\hline $7.77 \pm$ & $196.19 \pm$ & $99.79 \pm 13.05$ & $63.88 \pm$ \\
0.16 & 23.96 & & 4.88 \\
\hline
\end{tabular}

\subsection{Effect on vegetation growth using different approaches of plantation}

Vegetation being an important component for proper functioning of a $\mathrm{CW}$, it is necessary to monitor its growth so as to maintain aerobic pockets in the $\mathrm{CW}$ bed required by aerobic microorganisms for survival and consequent degradation of organic matter. The vegetation in $\mathrm{CBCW}$ as mentioned in section 2.4 showed improper growth pattern. Initially, the vegetation brought was used as they were plucked from the streams directly into the spaces created in the mesh of AC sheets. There was a possibility that by directly inserting plants in the small BR spaces the bulb portion of plant could have got damaged after reaching the bottom of BR leading to its early death. The second approach of plantation wherein the stem/leaves of the plants were tied together and kept suspended onto the edge of BR too wasn't a success as the roots were either not fully exposed to wastewater or flooded with wastewater due to fluctuations in wastewater depth owing to diurnal flow variation as recorded from flow monitoring. Generally in subsurface wetlands, water level is the most critical aspect of plant survival during the first year after planting. A common mistake is to assume that because the plant being a wetland plant, it can tolerate deep water. Frequently, too much water creates more problems for wetlands plants during the first growing season than too little because the plants do not receive adequate oxygen at their roots. [15] This validates large wilting of vegetation and subsequent dieoff. Also, using a thread for tying formed an incision on the leaves which subsequently damaged the entire leaf and after a certain time period, the entire plant as a whole. Amongst these methods, the final method of first acclimatizing the plants (subjected to serial dilution of wastewater) and then gripping them into perforated pipes of plastic bottles was found to be most effective. Vegetation used in this study was brought from nearby marshy lands and for their best survival as well as growth, small stalks (1 - 2 inches) during the first growing season in the substrate should only be kept in saturated conditions, not flooded. As the plants become well-established (2 - 3 months), water levels can be raised. Plants should be allowed to become well established prior to wastewater being introduced into the system since the plants need an opportunity to overcome stress after plantation before other stresses are introduced. A gradual rather than sudden increase in the concentration of the wastewater applied reduces shock to the vegetation. Most wetland species are adapted to daily or seasonal fluctuations in water level but most wetland plants can tolerate neither extended periods of flooding nor drying of their roots. [15]. Also, using perforated plastic bottle pipes ensured continuous supply of wastewater to roots as well as proper grip and space for roots to spread.

\subsection{Removal of organic matter in different plantation phases}

The $\mathrm{CBCW}$ was analyzed for its performance from August, 2018 to December, 2018. The total sample size accounts to 110 . As seen from Fig. 4, the removal of organic matter has an upward trend along with the time in months. It can be noted that COD removal in months of August and September, 2018 ranged from 17.85 \pm 5.51 $\%$ to $17.77 \pm 6.90 \%$ for a hydraulic loading rate (HLR) ranging between $10.29 \mathrm{~m}^{3} / \mathrm{m}^{2} . \mathrm{d}$ and $0.76 \mathrm{~m}^{3} / \mathrm{m}^{2} . \mathrm{d}$ and organic loading rate (OLR) of $0.17 \mathrm{kgCOD} / \mathrm{m}^{2} . \mathrm{d}$ and $2.89 \mathrm{kgCOD} / \mathrm{m}^{2}$.d. The removal is lower which could be due to vegetation in $\mathrm{CBCW}$ still in their initial stage of growth along with improper growing pattern in these months. Also, early wilting of vegetation required removal of decayed plants from BRC. This could have created void of aerobic pockets at root zones of the plants. This may have led to non-growth of aerobic microorganisms which are mainly related to degradation of organic matter. However, the months of September and October, 2018 has seen a steady spike in COD removal increasing by about $4 \%$ and $12 \%$ respectively. A similar trend was observed for $\mathrm{BOD}_{3}$ removal as well. This could be validated by the proper development of young shoots and root matrix of the bottled vegetation in $\mathrm{BRC}$. The root matrix as well as the brickbat media provide abundant space for microorganisms to thrive and aid in further COD removal. The maximum COD removal was found to be $37.5 \%$. There was a certain decrease in the removal efficiency in month of December, 2018. As the city of Sangli experiences cold winters in this month, the microbial activity is hampered due to low temperature which validates the drop in COD removal. Also, the lower COD removal can be validated by presence of residual $\mathrm{BOD}_{3}$ entering the effluent due to decay of dead roots, shoots or leaves left within the wetland bed unchecked. The average effluent COD and $\mathrm{BOD}_{3}$ observed was $130 \mathrm{mg} / \mathrm{L}$ and $60 \mathrm{mg} / \mathrm{L}$ respectively. An improvement in removal efficiency can be expected after proper and uniform growth of vegetation on the entire $\mathrm{CBCW}$ bed. 


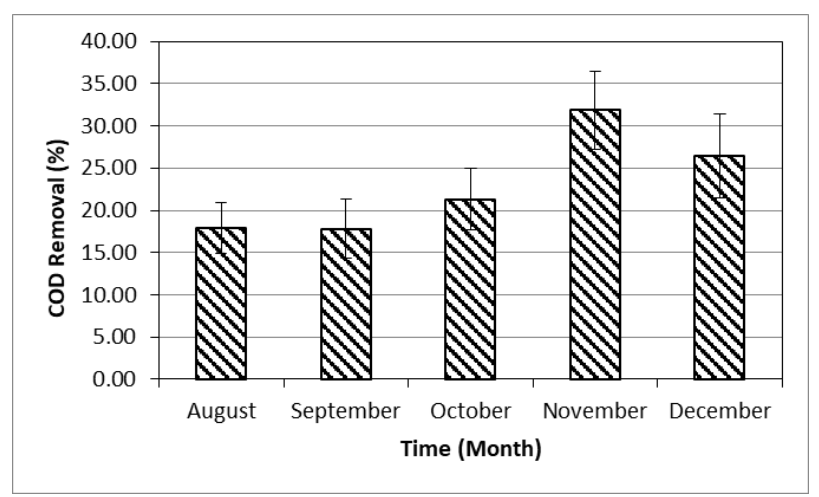

Fig. 4 Monthly variation in COD removal efficiency

\section{Conclusions}

The chemical characterization of wastewater reveals that biological treatment can be given to the anaerobically treated domestic wastewater and thus constructed wetland is a suitable alternative to conventional wastewater treatment systems. The problems faced while growing vegetation for adequate working of a Composite Biorack Constructed Wetland is highlighted in the present study by using different approaches. It is concluded that vegetation growth is majorly affected by improper suspension of vegetation into Biorack leading to damage to roots and bulbs of the plants as well as fluctuating flow of wastewater and wastewater level. It is also found that proper gripping of the vegetation in perforated plastic bottles and then suspending in BRs creates ample space for root development and growth of new shoots. Also, acclimatization of the new saplings in the present wastewater conditions plays an important role in vegetation growth. A maximum COD removal efficiency of $37.50 \%$ is achieved in $\mathrm{CBCW}$ with average effluent COD and BOD3 of $130 \mathrm{mg} / \mathrm{L}$ and $60 \mathrm{mg} / \mathrm{L}$ respectively. The values don't completely satisfy permissible land application wastewater standards set by $\mathrm{CPCB}$, India and thus a further tertiary treatment is required before its disposal. From the removal trend, it can be deduced that vegetation growth affects COD removal significantly. Decay of roots, shoots and wilted leaves left unchecked within the BRs can contribute to drop in COD removal efficiency. Hence, care should be taken while harvesting to remove such unwanted plant debris which can affect the performance of the system.

The present study is a part of research project sanctioned by Department of Science and Technology DST) under Water Treatment Initiative (WTI) programme. The authors sincerely acknowledge and thank DST for providing financial support to undertake this study.

\section{References}

1. S. Abou-Elela and M. Hellal, Ecol Engg, 47, 209-213, (2012)

2. S. Abou-Elela, G. Golinelli, A. El-Tabl and M. Hellal, Water Sci \& Tech, Vol. 69 (1), 38-47, (2014)
3. APHA, Standard Methods for the Examination of Water and Wastewater, American Water Works Association, Water Environment Federation (2012)

4. L. Cui, Y. Ouyang, Q Lou., F Yang., Y. Chen, W. Zhu and S. Luo, Ecol Engg, 36, 1083-1088 (2010)

5. D. Ghosh and B. Gopal, Ecol Engg, 36, 10441051 (2010)

6. S. Jamshidi, A. Akbarzadeh, K. Woo and A. Valipour, J. of Env Health Sci \& Engg, 12, 1-12 (2014)

7. L. Marchand, F. Nsanganwimana, N. Oustriere, Z. Grebenshchykova, K. Lizama-Allende, M. Mench, Ecol Engg, 64, 291-300 (2014)

8. Y. Patil and G. Munavalli, Ecol Engg, 95, 492500 (2016)

9. S. Sathe and G. Munavalli, J. of Water Process Engg, 28, 240-249 (2019)

10. A. Valipour, V. Raman and V. Ghole, Ecol Engg, 35, 1797-1803 (2009)

11. A. Valipour, V. Raman and V. Ghole, J. of Env Sci and Engg, 53(3), 281-288 (2011)

12. J. Vyazmal, Water 2010', 2, 530-549 (2010)

13. J. Wang, L. Zhang, S. Lu, X. Jin and S. Gan, J. of Env Sci. 24(6), 1006-1013 (2012)

14. A. Yadav, R. Abbassi, N. Kumar, S. Satya, T. Sreekrishnan and B. Mishra, Chem Engg J., 211, 501-507 (2012)

15. "Manual Constructed Wetlands Treating Municipal Wastewaters", United States Environmental Protection Agency, September 2000 